\title{
A 73,128 bp de novo deletion encompassing the OPN1LW/OPN1MW gene cluster in sporadic Blue Cone Monochromacy: a case report
}

\author{
Elena Buena-Atienza, Fadi Nasser, Susanne Kohl and Bernd Wissinger * (D)
}

\begin{abstract}
Background: Blue Cone Monochromacy (BCM) is a rare congenital cone dysfunction disorder with X-linked recessive mode of inheritance. BCM is caused by mutations at the OPN1LW/MW cone opsin gene cluster including deletions of the locus control region (LCR) and/or parts of the gene cluster. We aimed at investigating the clinical presentation, genetic cause and inheritance underlying a sporadic case of BCM.

Case presentation: We report a 24-year-old male presenting with congenital photophobia, nystagmus and colour vision abnormalities. There was no history of retinal dystrophy in the family. Clinical diagnosis of BCM was supported by genetic investigations of the patient and his family members. Molecular genetic analysis of the OPN1LW/OPN1MW gene cluster revealed a novel deletion of about $73 \mathrm{~kb}$ in the patient encompassing the LCR. The deletion was absent in the X-chromosomes of both the mother and transmitting grandfather.

Conclusions: The present report provides the clinical findings and the genetic basis underlying a sporadic BCM case which is caused by a de novo deletion within the OPNILW/MW gene cluster originating from the mother's germline due to Alu-repeat mediated recombination. This is the first report of a de novo deletion resulting in BCM, highlighting the importance to consider BCM and perform genetic testing for this condition in male patients with cone dysfunction also in the absence of a positive family history.
\end{abstract}

Keywords: De novo mutations, Sporadic cases, Blue Cone Monochromacy, Colour vision deficiency, Alu-mediated recombination, Retinal dystrophy

\section{Background}

Blue Cone Monochromacy (BCM; OMIM\#303700) is characterized by reduced visual acuity, photophobia, colour vision deficiency and it is regularly accompanied by congenital nystagmus and myopia [1]. The clinical findings in BCM patients largely overlap with those of achromatopsia patients. An X-linked recessive pattern of inheritance is a practical distinctive trait of BCM. Yet, lack of family history in simplex cases hinders prioritization of genetic tests. In addition, genetic testing for $\mathrm{BCM}$ is not regularly available at most diagnostic

\footnotetext{
* Correspondence: wissinger@uni-tuebingen.de

Institute for Ophthalmic Research, Centre for Ophthalmology, University of Tuebingen, Elfriede-Aulhorn 7, D-72076 Tuebingen, Germany
}

genetic testing laboratories due to the complexity of the opsin gene cluster.

The genes for the long wavelength sensitive (OPN1LW; LW opsin gene) and middle wavelength sensitive (OPN1MW; MW opsin gene) pigments in LW and MW cone photoreceptors are located on the X-chromosome and arranged as a head-to-tail tandem array in the so-called OPN1LW/OPN1MW gene cluster. The first position in the cluster is generally occupied by an OPN1LW gene copy followed by one or multiple OPN11MW gene copies. Each gene copy possesses a direct upstream promoter, while the expression of the genes in the gene cluster is regulated by an upstream locus control region (LCR). The LCR is a $0.6 \mathrm{~kb}$ cis-regulatory sequence essential to drive expression in a distance dependent manner so

(c) The Author(s). 2018 Open Access This article is distributed under the terms of the Creative Commons Attribution 4.0 International License (http://creativecommons.org/licenses/by/4.0/), which permits unrestricted use, distribution, and reproduction in any medium, provided you give appropriate credit to the original author(s) and the source, provide a link to the Creative Commons license, and indicate if changes were made. The Creative Commons Public Domain Dedication waiver (http://creativecommons.org/publicdomain/zero/1.0/) applies to the data made available in this article, unless otherwise stated. 


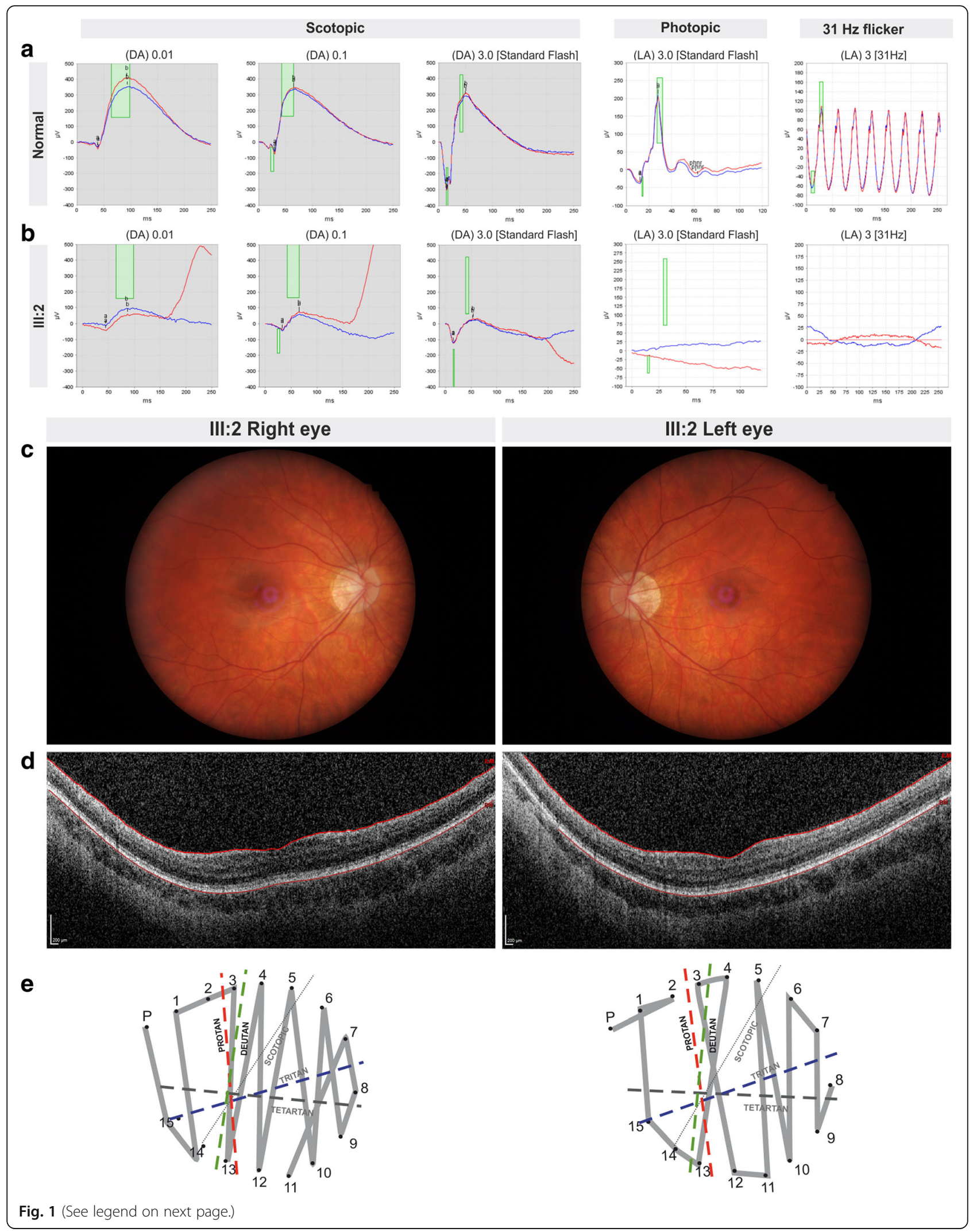


(See figure on previous page.)

Fig. 1 Ophthalmological findings in BCM patient III:2 a Full-field ERG recordings according to ISCEV Standards of a normal subject $\mathbf{b}$ ERG of the index patient (III:2) at last clinical examination (24 years old) showing mild reduction under dark-adapted (DA) scotopic conditions and undetectable responses under light-adapted $(\mathrm{LA})$ photopic responses in both eyes. Strength of the light stimulus is given in photopic $\mathrm{cd}^{*} \mathrm{~s} / \mathrm{m}^{2}$, if not stated otherwise. Grey sections indicate scotopic recording conditions. Red colour represents right eye and blue the left eye. Green rectangles indicate 5-95\% confidence intervals for amplitudes and implicit times of a normal population. c Right and left eye fundus image of the patient showing tilted optic disc. $\mathbf{d}$ Right and left eye OCT shows normal retinal architecture in the BCM patient with thinned photoreceptor layers. e Farnsworth Panel D-15 saturated shows confusion errors along the protan-deutan axes for both eyes

that essentially only the first two gene copies within the gene cluster are expressed [2].

Deletions affecting the OPN1LW/OPN1MW gene cluster on Xq28 have been estimated to account for $30-40 \%$ of all mutations found in BCM patients [3, 4]. Whereas deletions may be restricted to the LCR region, a number of deletions have been reported to extend towards the human cone opsin gene cluster, i.e. deleting partially or completely the OPN1LW and/or OPN1MW gene copies [1,3-7].

In this report, we present a male with a clinical phenotype of BCM carrying a novel deletion originated by a de novo mutation event in the OPN1LW/OPN1MW gene cluster. Herein, we describe the clinical findings of the patient, present the results from molecular genetic investigations of the patient and his family members, and discuss the putative underlying mechanism leading to this de novo mutation.

\section{Case presentation}

A 24-year-old male presented with photophobia since birth. No family history for colour vision defects or retinal dystrophies was reported. Myopia with an refractive error of - 5.50 D (right eye) and - 6.50 D (left eye) and astigmatism were found in the patient (III:2) at the age of 8 months along with nystagmus but devoid of strabismus. Glasses were given at the age of 1 year. Difficulties distinguishing colours were noticed by his parents at the age of 3 years. Achromatopsia was the first suspected diagnosis. At the age of 4 years occlusion therapy alternating in both eyes for 2 months was attempted to treat amblyopia but was unsuccessfull. A best-corrected visual acuity of 20/200 was measured with Snellen charts at the age of 6 years. No brain injuries were detected by magnetic resonance imaging. Visual evoked potential flash and B-scan ultrasonography performed normal for both eyes. At the age of 11 years a visual acuity of 20/ 250 was measured. At the latest exam at the age of 24 years, full-field light- and dark-adapted electroretinogram (ERG) recordings were performed according to the International Society for Clinical Electrophysiology of Vision (ISCEV) standard protocol [8]. Subnormal amplitudes under scotopic conditions and extinct responses under photopic conditions were observed in both eyes of the patient (III:2) in comparison to normal controls (Fig. 1a-b). A visual acuity of 20/400 was measured for both eyes with a myopic correction of $-12.00 \mathrm{D}$ (right eye) and $-11.50 \mathrm{D}$ (left eye). Anterior segment, pupillary reflexes and intraocular pressure revealed no abnormalities. Eye fundus examination revealed normal retinal vessels, optic nerve heads showing tilted optic discs with myopic conus and the maculae had elapsed reflex without waxy reflex (Fig. 1c). Spectral domain optical coherence tomography (SD-OCT) performed with Spectralis OCT (Heidelberg Engineering) showed normal retinal architecture with thinned photoreceptor layer (Fig. 1d). Colour vision test evaluated with the Farnsworth D-15 Colour Test revealed protan-deutan confusion errors (Fig. 1e).

Mutation screening of OPN1LW/OPN1MW gene cluster in the patient (III:2) was performed as previously described [9]. Genotyping PCRs with genomic DNA from the patient (III:2, see Fig. 2a for a pedigree of the genetically investigated family members) revealed absence of the LCR and promoter regions of both OPN1LW and OPN1MW genes - indicative for a large deletion - but presence of the 3' parts of the OPN1MW gene, namely exons 4, 5 and 6 . Upon fine sequence tag site content mapping, the deletion was finally bridged with a PCR amplicon performed with primers BCM\#27_F (5' - TCGACCCAGAATTAACCTC TCT -3') and BCM\#27BPR (5'-TCTAAAAATGGACA AGGATTAACCA -3') which was sequenced with Sanger to determine the exact breakpoints in patient III:2 (Fig. 2b and Fig. 2c). The deletion, NC_000023.11:g.154,118,184_154,191,311del, encompasses 73,128 bp with the centromeric breakpoint located in the intergenic region between $M E C P 2$ and $O P N 1 L W$ and the telomeric breakpoint within intron 3 of OPN1MW (Fig. 2d).

A sequence alignment of the breakpoint junction sequence in the patient (III:2) with the corresponding non-mutant sequence sections from his grandfather (I:1) revealed an overlapping stretch of $13 \mathrm{bp}$ between the centromeric and the telomeric breakpoint sequences (Fig. 2b) shared by two Alu elements. The sequence remnants embedded within the deletion breakpoints resembled the junction of two Alu elements; (1) a fossil right Alu momomer (FRAM) element at the centromeric breakpoint of the deletion, and (2) an AluJo element in intron 3 of OPN1MW at the telomeric breakpoint of the deletion (Fig. 2d). Microsatellite marker analysis revealed 


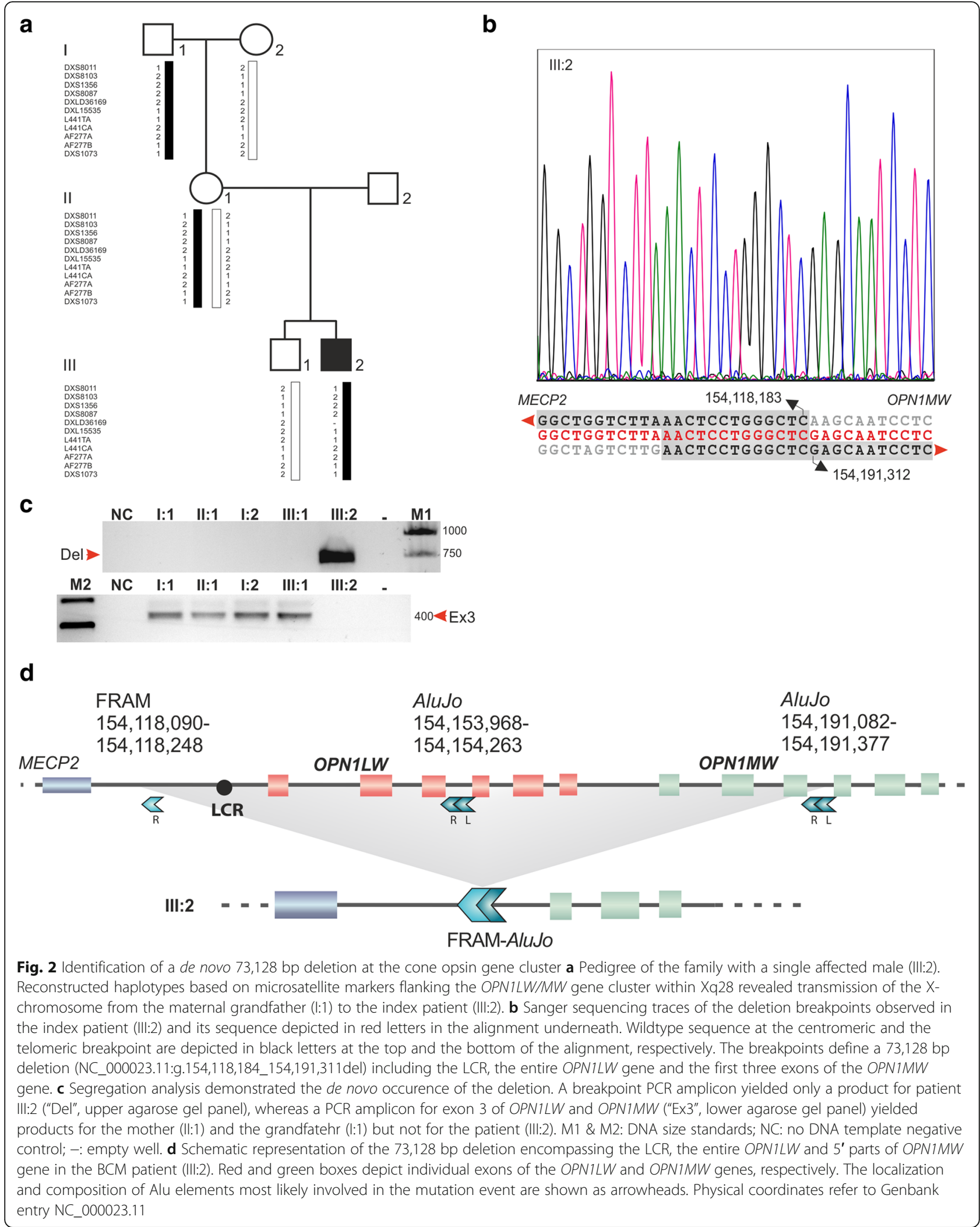


that the X-chromosome present in the patient (III:2) had been transmitted from his maternal grandfather (I:1, Fig. 2a). Segregation analysis performed by means of breakpoint PCR amplification showed that neither the patient's grandfather (I:1) nor the mother (II:1) carry the deletion (Fig. 2c). The CARE guidelines were followed in reporting this case.

\section{Discussion and conclusions}

In this report we describe a deletion spanning 73,128 bp within the human OPN1LW/OPN1MW cone opsin gene cluster in a male with clinical features fully compatible with BCM but lacking family history for this condition. The molecular findings were critical to establish the correct diagnosis for the patient. A more thorough clinical investigation at 24 years of age showed results concordant with a BCM phenotype (Fig. 1a-e). While there is evidence for a link between emmetropization and the $M$ to $\mathrm{L}$ cone ratio [10], the mechanism(s) underlying myopia in BCM are still unsolved. Presumably due to the high myopia and the lack of cone contribution to the dark-adapted responses, a mild reduction of scotopic signals was observed (Fig. 1a-b). Previously, rod responses to dim stimuli were reported to be in the lower normal or subnormal range in BCM patients $[4,6]$.

The NC_000023.11:g.154,118,184_154,191,311del mutation in this patient has not been previously reported. The de novo nature of the deletion was confirmed by haplotype reconstruction and segregation analysis of all family members available. While the X-chromosome present in the patient (III:2) had been transmitted from his maternal grandfather (I:1, Fig. 2a), the NC_000023.11:g.154,118,184_154,191,311del mutation was absent in the patient's grandfather (I:1) and mother (II:1, Fig. 2c). Therefore the mutation identified in the patient of this particular case represents a de novo event that occurred in the mother's germline (II:1, Fig. 2c).

The breakpoint sequences of the deletion are localized within Alu repetitive elements. Based on the sequence composition prior to deletion and the actual Alu sequence remnants found at the deletion breakpoints, it is tempting to propose a mechanism underlying this de novo mutation event. We hypothesize that the monomeric FRAM element underwent pairing with the left arm of the bipartite AluJo element and induced subsequent recombination within the $13 \mathrm{bp}$ of microhomology. FRAM and AluJo most likely promoted the origin of the deletion during meiosis through misalignment of the homologous stretch [11]. The FRAM and AluJo elements may have acted in this very event most likely as nucleation spots allowing mispairing and making the sequence prone to homologous recombination [11]. The resulting deletion gave rise to a hybrid FRAM-AluJo element. Notably, more than $95 \%$ of the Alu-mediated deletion events identified in the human genome occurred as Alu-mediated unequal homologous recombination [12]. According to the aforementioned model proposed, the Alu element mediated illegitimate recombination may have occurred either by mispairing of sister or non-sister chromatids (interchromosomal), or between elements on the same chromosome (intrachromosomal).

Although several studies have previously reported deletions including the LCR to be associated with a BCM phenotype [1, 3-7], this is the first de novo deletion reported to underlie a BCM phenotype. We have previously described a de novo intrachromosomal gene conversion event in the male germline transferring a pathogenic haplotype from OPN1MW to OPN1LW [12]. Notwithstanding, the BCM patient's grandfather presented with a linked colour vision deficiency due to a pathogenic haplotype in OPN1MW and the patient's mother was a carrier of the converted OPN1LW gene [13]. Our case report herein describes a BCM patient bearing a unique mutation that stem from an independent de novo event in a family with no history of BCM or colour vision abnormalities.

In summary, we were able to elucidate the origin of a newly emerged mutation in an isolated case of BCM. These findings emphasize the need to test for X-linked $\mathrm{BCM}$ in cases with a clinical diagnosis of cone dysfunction, even in the absence of a family history in order to obtain a correct clinical diagnosis and a valid basis for proper genetic counselling. The novel 73,128 bp deletion within the OPN1LW/MW gene cluster occurred most likely as a result of an Alu recombination-mediated mechanism. This is the first report of a de novo deletion resulting in $\mathrm{BCM}$.

\section{Abbreviations}

BCM: Blue Cone Monochromacy; ERG: Electroretinogram; FRAM: Fossil right Alu momomer; LCR: Locus control region; SD-OCT: Spectral domain optical coherence tomography

\section{Acknowledgements}

We thank Britta Baumann for excellent technical assistance. We further acknowledge support by the Deutsche Forschungsgemeinschaft and the Open Access Publishing Fund of the University of Tübingen.

\section{Funding}

This work was supported by the European Union's Seventh Framework Programme for research, technological development and demonstration [grant agreement no 317472] and by the BCM Families Foundation.

\section{Availability of data and materials}

The mutation has been deposited and stored publicly available in ClinVar under the link https://www.ncbi.nlm.nih.gov/clinvar/variation/448956/ with accession number SCV000612182.

\section{Authors' contributions}

FN collected clinical data. EB-A performed the genetic investigations and summarized molecular data. SK managed the family case. BW provided the study concept and design. All authors wrote, read and approved the final manuscript. 


\section{Ethics approval and consent to participate}

All procedures performed involving human participants were in accordance with the tenets of the WMA Declaration of Helsinki and have been approved by the Ethics Committee of the Medical Faculty, Eberhard-Karls University Tuebingen.

\section{Consent for publication}

Written informed consent was obtained from the patient to publish the medical information supplied in this case report.

\section{Competing interests}

The authors declare that they have no competing interests.

\section{Publisher's Note}

Springer Nature remains neutral with regard to jurisdictional claims in published maps and institutional affiliations.

Received: 15 January 2018 Accepted: 12 June 2018

Published online: 26 June 2018

\section{References}

1. Nathans J, Davenport CM, Maumenee IH, Lewis RA, Hejtmancik JF, Litt M, et al. Molecular genetics of human blue cone monochromacy. Science. 1989; 245(4920):831-8.

2. Winderickx J, Battisti L, Motulsky AG, Deeb SS. Selective expression of human X chromosome-linked green opsin genes. Proc Natl Acad Sci U S A. 1992;89(20):9710

3. Nathans J, Maumenee $I H$, Zrenner E, Sadowski B, Sharpe LT, Lewis RA, et al. Genetic heterogeneity among blue-cone monochromats. Am J Hum Genet. 1993;53(5):987-1000.

4. Ayyagari R, Kakuk LE, Bingham EL, Szczesny JJ, Kemp J, Toda Y, et al. Spectrum of color gene deletions and phenotype in patients with blue cone monochromacy. Hum Genet. 2000;107(1):75-82.

5. Ayyagari R, Kakuk LE, Coats CL, Bingham EL, Toda Y, Felius J, et al. Bilateral macular atrophy in blue cone monochromacy (BCM) with loss of the locus control region (LCR) and part of the red pigment gene. Mol Vis. 1999;5:13.

6. Kellner U, Wissinger B, Tippmann S, Kohl S, Kraus H, Foerster MH. Blue cone monochromatism: clinical findings in patients with mutations in the red/ green opsin gene cluster. Graefes Arch Clin Exp Ophthalmol. 2004;242(9): 729-35.

7. Gardner JC, Liew G, Quan Y-H, Ermetal B, Ueyama H, Davidson AE, et al. Three different cone Opsin gene Array mutational mechanisms with genotype-phenotype correlation and functional investigation of cone Opsin variants. Hum Mutat. 2014:35(11):1354.

8. McCulloch DL, Marmor MF, Brigell MG, Hamilton R, Holder GE, Tzekov R, et al. ISCEV standard for full-field clinical electroretinography (2015 update). Doc Ophthalmol. 2015;130(1):1-12.

9. Cideciyan AV, Hufnagel RB, Carroll J, Sumaroka A, Luo X, Schwartz SB, et al. Human cone visual pigment deletions spare sufficient photoreceptors to warrant gene therapy. Hum Gene Ther. 2013;24(12):993-1006.

10. Gisbert $\mathrm{S}$, Schaeffel F. M to $L$ cone ratios determine eye sizes and baseline refractions in chickens. Exp Eye Res. 2018;172:104-11.

11. Batzer MA, Deininger PL. Alu repeats and human genomic diversity. Nat Rev Genet. 2002;3(5):nrg798.

12. Liu GE, Alkan C, Jiang L, Zhao S, Eichler EE. Comparative analysis of Alu repeats in primate genomes. Genome Res. 2009;19(5):876.

13. Buena-Atienza E, Rüther K, Baumann B, Bergholz R, Birch D, Baere ED, et al. De novo intrachromosomal gene conversion from OPN1MW to OPN1LW in the male germline results in blue cone Monochromacy. Sci Rep. 2016;6: 28253.

Ready to submit your research? Choose BMC and benefit from:

- fast, convenient online submission

- thorough peer review by experienced researchers in your field

- rapid publication on acceptance

- support for research data, including large and complex data types

- gold Open Access which fosters wider collaboration and increased citations

- maximum visibility for your research: over $100 \mathrm{M}$ website views per year

At BMC, research is always in progress.

Learn more biomedcentral.com/submissions 